\title{
Education on Qualified Interpreters-Strategies of Cultivation on Psychological Quality
}

\author{
ZHANG Jinchen, WANG Lin \\ University of Shanghai for Science and Technology, Shanghai, China
}

\begin{abstract}
In the modern era, educators have to attach importance on the requirements of talent quality training in the 21st century and make unremitting efforts to meet these requirements. How to help students overcome state fright and adapt to the actual scene of interpreting has been a major concern for professors and teachers. Psychological quality, which is as important as other interpretation skills, such as listening, is a premise of a successful and qualified interpretation. This essay will analyze how to educate a qualified interpreter in terms of psychological quality by various aspects, including putting more emphasis on bilingual learning, enriching students' cultural connotations, enhancing teachers' comprehensive quality, cultivating students' English public speaking ability, creating authentic interpretation environments, improving students' adaptability of different interpretation environments, and using other practical skills.
\end{abstract}

Keywords: education on English interpretation, psychological quality, interpretation teaching strategies

\section{Introduction}

Under the background of globalization, the training of interpreters is of great significance both to the promotion of political, economic, and cultural exchanges and to the cooperation between China and foreign countries. In 1979, the Ministry of Education released Teaching Plan for English Majors in Colleges and Universities and Practical Teaching Syllabus for the Basic Stage of English Majors. Since then, interpretation course has been subsumed into the compulsory course for English major in almost all colleges and universities in China. During the process of interpretation education, psychological quality serves as an important role to the quality of interpretation. According to self-schema theory in psychology, negative possible self is associated with anxiety, which may reduce the level of performance. A number of experiments have shown the fact that compared with professional interpreters, student interpreters are more susceptible to interpretation anxiety, resulting in a decline in the quality of interpretation (Wang, 2018, p. 71). Unsatisfactory psychological qualities may directly exert a negative impact on interpretation performance in terms of accuracy, fluency, and integrity. Therefore, a qualified interpreter is required to have excellent psychological qualities, which have already become industry standards. With the same skills and levels of language, interpreters who are good at adjusting their psychological quality can perform successfully or outperform expectations, while vice versa. Improving

\footnotetext{
Supported by grants of University of Shanghai for Science and Technology. Project number: WYJYPY001 and SK17ZD06. ZHANG Jinchen, undergraduate, College of Foreign Languages, University of Shanghai for Science and Technology, Shanghai, China.

WANG Lin, Doctor's degree, Associate Professor, College of Foreign Languages, University of Shanghai for Science and Technology, Shanghai, China.
} 
psychological quality tends to significantly enhance interpretation quality and meet audience satisfaction. Here are several strategies that can be used in interpretation education to improve psychological quality.

\section{Put More Emphasis on Bilingual Learning}

Focusing on bilingual learning at the same time can reduce the possibility of unexpected situations caused by inaccurate expression to some extent. Once this happens, the interpreter who has accepted adequate bilingual learning training will be no longer anxious and his or her psychological quality will be better, leading to a more successful performance. Interpretation means transformation between two languages. During the interpreting training, both Chinese and English should be taken seriously as it is impossible to interpret successfully if one only focuses on English learning, but ignores Chinese learning. However, due to advanced networking technology in this modern era, Chinese people, especially youngsters are accustomed to using internet slangs instead of formal language in our daily lives. Consequently, if an interpreter has a good command of English but is poor at Chinese, he or she is not able to use accurate, precise, and refined Chinese words to interpret them even though he or she can fully comprehend the deep meaning behind sentences. In order to cultivate qualified interpreters who are regarded as "language experts", teachers should impart students how to interpret with formal expressions and exercise their bilingual competence because good bilingual competence is the foundation of composure, which can help interpreters to alleviate anxiety to some extent under various situations in interpretation.

\section{Enrich Students’ Cultural Connotations}

As the cross-cultural challenges encountered by students are often invisible barriers to fluctuate their psychological conditions, having a clear understanding of distinctions of various cultures is a key factor to ensure good quality of an interpretation. Interpretation is a translational activity in which one produces a first and final translation on the basis of a one-time exposure to an expression in a source language. Interpretation is not so much a simple transformation between two different languages as a communication or exchange of different cultures between two nations or ethics. Every nation has its own unique development history, traditions, lifestyles, rituals, values, etc. It is generally known that China has a long history, stretching back thousands of years and there are numerous historical figures, such as the Forbidden City, the Great Wall, while western countries have their own historical figures, such as Shakespeare, the poet who emerged in the renaissance. During interpretation, if the interpreter simply translates the names of relevant historical figures and events directly without any explanation, the recipient of information would be puzzled and confused, which will lead to the undesirable consequence that important information would not be conveyed successfully. Under some specific circumstances, for example, when interpreting foreign food and lifestyles, interpreters are no doubt faced with some challenges and difficulties as some words or phrases are untranslatable in interpretation, and then it is necessary and essential for them to further explain different cultures of the two regions or countries by digging the deep meaning behind these words and to express them in a simple way. For example, in the north of China, people will make fire by utilizing a special stove called "Kang", but there is no exact western word to explain "Kang”. In this way, if the translator only translates literally, the recipient will not make them out at all. Therefore, the schools of English in every universities or colleges should attach more importance to imparting knowledge and skills that help students to understand cultural differences which exist in ones' own country and in others and handle them correctly during interpretation. This end can be achieved 
by watching some foreign movies or documentaries in the class or by communicating with foreign exchange students. The most important point is that students should be taught to have a sense of consciousness to interpret the meaning behind the cultural differences instead of interpreting directly under these circumstances. What's more, it is particularly important for teachers to have a high level of cultural accomplishment. Teachers who are expected to play a role more than a "knowledge porter" and to shoulder the responsibility of cultivating students' cross-cultural awareness are required to have a thorough and meticulous understanding of the cultural traditions, customs, humanities, and history of different countries in order to create learning environment for students. Only by penetrating cross-cultural awareness throughout daily teaching can students own good psychological quality and have a normal performance during interpretation.

\section{Enhance Teachers' Comprehensive Quality}

In China, psychological quality of most of the interpretation teachers is not open enough. Due to the situation of our country and the uneven levels of teacher, many of them are still be trapped into the obsolete teaching modes as they are hardly able to discover new teaching methods to teach students effectively. Teachers should play a role as a beacon light in students' education rather than a stumbling block for children. Teachers should be required to have advanced teaching ideas and be able to train students' courage through a series of teaching methods. For example, a Japanese school asks students to do some embarrassing behaviors, such as wearing a suit and singing loudly in the busy street in order to train their courage. This case shows that students can be more confident and overcome stage fright through singing in crowded places such as teaching buildings or canteens. It appears that an open-minded and creative teacher with high comprehensive quality is more likely to cultivate a qualified interpreter as students' psychological quality can be enhanced dramatically through multiple methods of teaching.

\section{Cultivate English Public Speaking Ability}

English public speaking is an essential mean to exercise communicative ability, logical thinking ability, and written expression ability. If one or two students are forced to give a lecture to express their views logically and artistically towards different situations, different groups of audience, different themes or time in front of the students and teachers at the end of each course, they will have an easy access to understand different speech styles and learn how to write speeches as well as directly improve their language expressive ability in both theory and practice. Teachers had better demand that speeches given by students should have complete and rich content, rigorous structure, correct grammar, appropriate expression, in order to encourage students to achieve the end of fluent topic-setting speeches and impromptu speeches in English without being affected by outside world even under high pressure environment. In this way, students tend to have a balanced communication ability and lay a solid foundation for their good psychological quality of interpretation.

\section{Create Authentic Interpretation Environments}

Classroom environment plays a significant role in the teaching quality of interpretation course as it may affect the learning effect of students to some extent (Zhou, 2019, p. 106). Thus, a stimulated interpreting environment, giving students a sense of anxiety and oppression, is an effective mean to exercise interpreters' psychological quality. Most of the interpreting classes in our nation are dominated by teachers, but the undesirable consequences of inactive involvement of students in classroom performance are always be 
neglected. One of the most practical measures is to simulate an authentic environment that restores the authenticity and practicality of interpreting. In this way, students are more likely to embrace enthusiasm and motivation for learning and to have a tendency to get less nervous during the process of interpretation. In a stimulated classroom atmosphere, students tend to be encouraged to remove his or her limits and then achieve self-breakthrough during the interpretation. The interpretation scenes, including meeting scenes, business negotiation scenes, press conferences, etc., allow learners to have a full understanding of the whole process of interpreting, so that they can broaden their horizons, accumulate interpretation experiences, find out their shortcomings, and train interpretation skills specifically. Through this kind of thorough training, students are less likely to be influenced by the psychological fluctuation when they are working in a real interpretation communication in the future.

\section{Improve Students’ Adaptability of Various Interpretation Environments}

In order to cultivate an interpreter with strong psychological quality, teachers should consciously create some urgent and extreme situations to test students' psychology quality and encourage students to overcome difficulties and continue to interpret with their own perseverance instead of giving up thoroughly even if they miss spokesman's key information. There is no doubt that interpreters have to deal with several daunting challenges and difficulties under different interpretation situations. A qualified interpreter is expected to adapt to the specific interpreting environments as quick as possible and respond immediately when they are on the spot. However, only few schools of English put an emphasis on education of adaptability for students, mainly because they do not realize that these seemingly little details may eventually exert a negative influence on students' psychology and affect their state and performance of interpretation. After adequate training in adaptability, interpreters are more likely to handle emergencies calmly and confidently.

\section{Utilize Other Practical Skills}

Even though people have a deep language foundation and make full preparations, their performances can also be easily affected by fluctuations in mentality. It is inevitable and unavoidable that people may be influenced easily by outside environment. In fact, there are multiple practical skills, such as breathing deeply or listening to light music that can be utilized to alleviate anxiety and help interpreters to focus attention. From a psychological point of view, positive psychological hints have a positive impact on people's psychological behavior. Positive words and sentences are considered as important implications for the brain of human being, while negative self-suggestion can have great side effects on people. Therefore, it is suggested that mental counselor in universities or colleges should be asked to teach students how to apply self-suggestive training or cognitive regulation training to calm themselves down. Here are some suggestions given by Journal of Chinese Health Psychology that can be used for students when they suffer from angst: They should give themselves a smile as smile can dispel anxiety and worry and bring happiness and self-confidence as well. Having a big smile on the face is equivalent of giving oneself a positive hint. Secondly, design some inspiring verbal expressions, such as "I am super confident”, which will impact one's psychology positively. Thirdly, students should be taught to confront failure directly. Especially during oral communication training, students without self-confidence are more likely to be afraid of being looked down by others and to escape from stiff competition unconsciously. In a nutshell, suggestions like these may help students overcome mental barriers, build self-confidence gradually, and perform better during interpretation. 


\section{Conclusion}

To summarize, there is a correlation between psychological quality of interpreters and interpretation quality. Professional and systematic training of psychological quality should never be ignored in the field of education. If students want to be qualified professional interpreters, it is essential and necessary for them to exercise their psychological quality. To achieve this end, universities and colleges should put more emphasis on bilingual learning, enrich students' cultural connotations, enhance teachers' comprehensive quality, cultivate students' English public speaking ability, create authentic interpretation environments, improve students' adaptability of different interpretation environments, and use other practical skills.

\section{References}

Luo, Y. T. (2018). Training strategies of students' psychological quality in English interpretation teaching. Theory and Practice of Education, 38(30), 53-54.

Wang, L. (2018). The impact of cognitive psychology on interpretation quality—a contrastive study of professional and student interpreters. Journal of Huaibei Normal University (Philosophy and Social Sciences Edition), 39(5), 71-77.

Zhou, S. Y. (2019). An investigation of university interpreting classroom environment. Journal of Changchun University, 29(4), 106-110. 